\title{
Paying attention to working memory: Similarities in the spatial distribution of attention in mental and physical space
}

\author{
Muhammet Ikbal Sahan $^{1}$ • Tom Verguts ${ }^{1}$ - Carsten Nicolas Boehler ${ }^{1} \cdot$ Gilles Pourtois $^{1}$. \\ Wim Fias ${ }^{1}$
}

Published online: 24 December 2015

(C) Psychonomic Society, Inc. 2015

\begin{abstract}
Selective attention is not limited to information that is physically present in the external world, but can also operate on mental representations in the internal world. However, it is not known whether the mechanisms of attentional selection operate in similar fashions in physical and mental space. We studied the spatial distributions of attention for items in physical and mental space by comparing how successfully distractors were rejected at varying distances from the attended location. The results indicated very similar distribution characteristics of spatial attention in physical and mental space. Specifically, we found that performance monotonically improved with increasing distractor distance relative to the attended location, suggesting that distractor confusability is particularly pronounced for nearby distractors, relative to distractors farther away. The present findings suggest that mental representations preserve their spatial configuration in working memory, and that similar mechanistic principles underlie selective attention in physical and in mental space.
\end{abstract}

Keywords Spatial attention · Working memory $\cdot$ Mental representations · Distractor confusion · Distribution · Orienting

Electronic supplementary material The online version of this article (doi:10.3758/s13423-015-0990-5) contains supplementary material, which is available to authorized users.

Muhammet Ikbal Sahan

muhammet.sahan@ugent.be

1 Department of Experimental Psychology, Ghent University, Henri Dunantlaan 2, B-9000 Ghent, Belgium
Humans constantly form mental representations of the external world and keep them active in working memory for further cognitive elaboration. Recent accounts of working memory have described the process of actively maintaining mental representations as selective attention directed toward internal representations (Awh \& Jonides, 2001; Oberauer, 2009; Postle, 2006). Moreover, it has been claimed that selective attention directed toward internal representations depends heavily on resources that are shared with selective attention directed toward external stimuli (Kiyonaga \& Egner, 2013). Accordingly, a growing number of studies have shown that orienting attention in mental space is characterized by the same behavioral patterns and recruits the same neural systems as orienting attention in the external world (Gazzaley \& Nobre, 2012).

Most studies investigating the orienting of attention in working memory have used a variant of the retro-cueing paradigm developed by Griffin and Nobre (2003). This paradigm closely matches the attentional pre-cueing paradigm (Posner, 1980) and allows for a direct comparison of the effects of attentional orienting in sensory and representational space. During the retention interval over which a visual array has to be remembered, a retro-cue is presented that points toward a location in the array. Subsequently, a probe is presented at a location in the array, and participants have to indicate whether the probe matches the item that was displayed at that location. As with pre-cues, cue validity effects are observed (facilitation when the retro-cue points to the location of the probe, and interference when a different location is cued), suggesting that attention has shifted to the cued location in mental space.

An important remaining question is how exactly information processing at the attended location is prioritized, as compared to unattended information. Although the field has largely accepted the view that attention operates similarly in working memory and perception, studies have mainly shown this in 
terms of the orienting of attention (Awh \& Jonides, 2001; Gazzaley \& Nobre, 2012); it is not known whether the mechanisms of attentional selection also show similar characteristics in mental and in physical space. A study of how attention is distributed across distractor locations might provide direct evidence for similar selection mechanisms in mental and physical space. The present article addresses this unexplored issue.

We directly compared the characteristics of the spatial distribution of attention in mental and in physical space under highly comparable conditions, by investigating interference from unattended locations. If there are similar attentional selection mechanisms for both mental and physical spaces, then the distribution characteristics in physical space should also apply to mental space. For this purpose, we developed a variant of the pre- and retro-cueing paradigm (Griffin \& Nobre, 2003). Crucially, we manipulated the distance between the attended location and the location at which the proposed probe was originally presented. We investigated how this distance determined the accuracy of rejecting a distractor probe (i.e., a probe not occurring at the indicated location). The distance functions induced by pre- and retro-cueing were compared.

Although it is generally agreed that attention enhances processing at the focus of attention relative to the unattended locations, theories differ with respect to how exactly attention is divided across the unattended locations. The spotlight (Posner, 1980) and zoom lens (Eriksen \& Yeh, 1985) models assume a focal, all-or-nothing attentional distribution, with a sharp boundary between information inside versus outside the focus of attention. These models predict no effect of distance. The gradient model (Downing \& Pinker, 1985) assumes a monotonic distribution, with activation decreasing with increasing distance from the focus of attention. This model predicts that distractors close to the focus of attention should be more difficult to reject than more remote distractors. Finally, center-surround models (Hopf et al., 2006; Störmer \& Alvarez, 2014; Tsotsos et al., 1995) postulate a nonmonotonic distribution, with enhancement turning into inhibition for the area surrounding the focus of attention. This inhibition then gradually diminishes with farther distance. Consequently, a nonmonotonic effect of distance is predicted, with the highest level of distractor activation being at distances far away from the target.

\section{Experiment 1}

\section{Method}

Participants Nineteen Ghent University students (16 females, three males; $M=23.7$ years old, $S D=5.24)$ participated in return for financial compensation. One participant was excluded from the analysis because of performance close to chance level (55.5\%). The research complied with the guidelines of the Independent Ethics Committee of the Department of Psychology and Educational Sciences of Ghent University. All participants gave written informed consent.

Task and design Participants viewed a stimulus array composed of five colored discs that were placed on an imaginary circle. They had to memorize the discs in order to make a delayed decision about a probe that occupied one of the five locations in this stimulus array. Participants had to decide whether the color of the probe stimulus presented at a certain location matched the color of the item from the stimulus array (Fig. 1). The probability of match trials was $50 \%$. In the case of a nonmatch, the color was randomly chosen from among the other four locations of the stimulus array. The nonmatch trials allowed us to study the distribution of attention across the stimulus array by comparing the distance effects between the probed location and the location from which the color was drawn (distances 1, 2, 3, and 4). Stimuli were presented unilaterally in either the upper left or the upper right quadrant, with $50 \%$ probability each. Two cue type conditions were used that only differed with regard to the time at which they appeared in the trial sequence. In the pre-cue condition, the cue was presented before the stimulus array, whereas in the retro-cue condition the cue was presented after the stimulus array. The probe appeared at the cued location in $80 \%$ of the trials (valid cue), whereas in $20 \%$ of the trials the probe appeared at a location other than the cued location (invalid cue).

Stimuli and procedure A white exclamation mark (!) announcing a new trial was presented against a black background in the middle of the screen for $500 \mathrm{~ms}$. This was followed by a random interval ranging from 400 to $600 \mathrm{~ms}$. In the pre-cue blocks, a white pre-cue was presented 1,500-2, $500 \mathrm{~ms}$ before the stimulus array for $100 \mathrm{~ms}$, whereas in the retro-cue trials, a fixation cross $\left(0.34^{\circ} \times 0.34^{\circ}\right.$ of visual angle $)$ was presented instead for $100 \mathrm{~ms}$. The stimulus array of five discs then appeared for $250 \mathrm{~ms}$. The discs (radius $0.5^{\circ}$ ) were placed on an imaginary circle of $4^{\circ}$ eccentricity. This configuration resulted in a $0.34^{\circ}$ tangential distance between the edges of neighboring discs. The colors (red, green, blue, yellow, and pink) were randomly assigned to the discs. In retro-cue blocks, a white retro-cue was presented 1,500-2, $500 \mathrm{~ms}$ after the stimulus array for $100 \mathrm{~ms}$, whereas in pre-cue trials, a white fixation cross instead was presented for $100 \mathrm{~ms}$. Cues were administered in a noncolored array by highlighting the circumference of one of the five discs, which were simultaneously presented in white. After another random interval ranging from 500 to $1,000 \mathrm{~ms}$, one of the five locations in the empty array was randomly probed for $100 \mathrm{~ms}$. A color was randomly drawn from the disc locations of the stimulus array in the nonmatch trials, whereas in match trials the color of the 
a Experiment $1 \& 2$

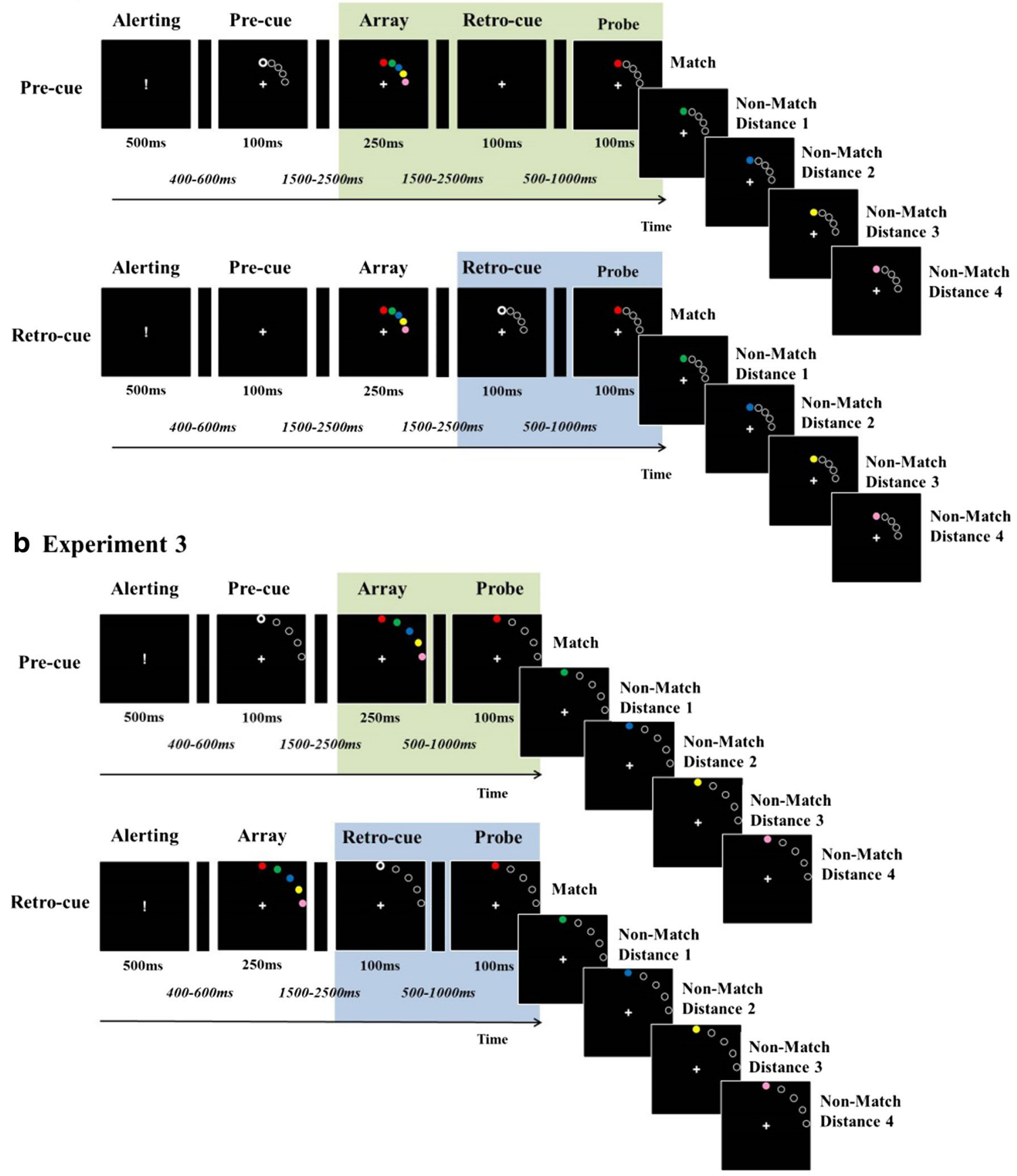

Fig. 1 Experimental paradigm. Participants memorized a stimulus array of colored discs in order to make a delayed decision about a probe (i.e., a delayed match-to-sample task). The task was to decide whether the probe stimulus matched the stimulus at the location in the original array. The task in Experiment 2 was the same as in Experiment 1, except for the spacing between the discs (a). The task in Experiment 3 was the same as in Experiment 2, except for timing differences in the trial sequences: The

probe corresponded to the color of that disc in the stimulus array. A 1,600-ms response deadline was imposed.

The pre- and retro-cue blocks of the task were tested in different sessions on two consecutive days, with the order counterbalanced across participants. Each session comprised
1,500- to 2,500-ms intervals before and after the stimulus array, respectively, in retro-cue and pre-cue conditions were removed (b). The different shaded areas indicate the time intervals between attentional selection and response execution in the pre-cue and retro-cue conditions. Notice that the period in the pre-cue condition of Experiments 1 and 2 was shortened in Experiment 3 to match the duration in the retro-cue condition. Italicized ranges of times relate to the interstimulus intervals

400 trials and lasted for $1 \mathrm{~h}$. Each session was divided into 16 blocks of 25 trials. Responses were registered via a Cedrus RB-730 response box with the index fingers of the left and right hands, with response mappings counterbalanced across participants. The instructions emphasized both speed and 
accuracy. Participants were informed about the dependency between the cue, stimulus, and probe arrays.

Data analysis Mean accuracies and reaction times in the nonmatch condition with valid cues were analyzed using a repeated measures analysis of variance (ANOVA) with Cue Type (pre- or retro-cue) and Distance (1 to 4) as withinsubjects factors. Reaction times below and above $2.5 \mathrm{SDs}$ from the means were discarded. Multivariate test results for the repeated measures are reported. To quantify the distance effect, regression analyses were performed to test for linear effects of distance conditional on cue type (following Lorch \& Myers, 1990). Differences in slopes between the cue types were tested by two-tailed paired $t$ tests. An alpha level of .05 was applied, and Bonferroni correction was used on multiple tests to control for false positives.

To exclude the possibility that the results were driven by the largest distances, which are limited to the outer edges of the array, we repeated all analyses with distance 4 excluded. Very similar results were obtained, so we do not report these analyses.

Additional analyses were performed as a manipulation check for attentional cueing in working memory (see the supplementary material).

\section{Results}

Accuracy The analysis revealed a main effect of Cue Type $\left[F(1,17)=13.683, p=.002, \eta_{\mathrm{p}}{ }^{2}=.446\right]$, with higher accuracies in pre-cue than in retro-cue trials. Furthermore, a main of effect of Distance $\left[F(3,15)=26.530, p<.001, \eta_{p}{ }^{2}=.841\right]$ was obtained. The analysis also revealed a significant Distance $x$ Cue Type interaction $\left[F(3,15)=4.217, p=.024, \eta_{\mathrm{p}}{ }^{2}=.458\right]$. Crucially, the regression analyses revealed an accuracy increase with increasing distance in both the retro-cue condition $($ slope $=5.57, S E=0.8), t(17)=6.965, p<.001,95 \% \mathrm{CI}=$ $[3.61,7.54]$, and the pre-cue condition (slope $=3.4, S E=$ $0.51), t(17)=6.635, p<.001,95 \% \mathrm{CI}=[2.14,4.66]$, although the increase was larger in the retro-cue than in the pre-cue condition, $t(17)=-2.43, p=.026,95 \% \mathrm{CI}=[-4.05,-0.29]$. See Fig. 2.

Reaction times The analyses only revealed a significant Distance $\times$ Cue Type interaction $\left[F(3,15)=6.464, p=.005, \eta_{\mathrm{p}}{ }^{2}=\right.$ $.564]$. The regression analyses showed that whereas reaction times remained stable in the retro-cue trials (slope $=3.1, S E=$ 4.23), $t(17)=0.728, p=.954,95 \% \mathrm{CI}=[-7.3,13.5]$, they decreased with distance in the pre-cue trials (slope $=-18.7, S E$ $=5.41), t(17)=-3.47, p=.006,95 \% \mathrm{CI}=[-32,-5.4]$. The decrease in reaction times in the pre-cue condition significantly differed from the pattern in the retro-cue condition, $t(17)=-$ $3.205, p=.005,95 \% \mathrm{CI}=[-36.17,-7.5]$

\section{Discussion}

The main purpose of this experiment was to study how attention is distributed across mental space and to directly compare this to the attentional distribution in physical space. Our results showed a clear effect of distance in physical space, in the sense that items that were located close to the target location were rejected with more difficulty (slower and less accurate) than were items located farther away. Using retro-cues, the same pattern was observed. This suggests that the mechanisms of attentional prioritizing are similar for mental and physical space.

However, two issues have to be dealt with before we can accept this conclusion. First, since the items in the visual arrays were densely spaced, it is possible that the observed effects emanate from crowding rather than from attentional selection. Second, the distance-related gradual performance differences in the retro-cue condition were only observed for accuracy, but not for reaction times. These issues will be explored further in Experiments 2 and 3, respectively.

\section{Experiment 2}

In principle, the findings of Experiment 1 could have emerged from visual crowding as a result of the dense configuration of the discs covering a small portion of the visual field (Levi, 2008). Indeed, when neighboring items are spaced at less than one tenth of the eccentricity at which they are presented, the discriminability of individual discs may become degraded (Levi, 2008). With an eccentricity of $4^{\circ}$ and an interitem gap of $0.34^{\circ}$, the visual arrays of Experiment 1 fell within this critical range. To rule out a crowding effect, we repeated Experiment 1 with an interitem spacing that was clearly above the crowding threshold.

\section{Method}

Participants Twenty other Ghent University students (18 female, two males; $M=19.6$ years old, $S D=2.4$ ) participated for course credits. One participant was excluded from the analysis because of performance close to chance level (46.5\%).

Stimuli, design, and procedure The parameters were the same as in Experiment 1, except that the spacing between the discs was increased. The tangential distance between the discs in the array was set to $1.50^{\circ}$ by increasing their eccentricity to $8^{\circ}$. These parameters clearly exceed the critical spacing measures $(\sim 0.1 \times$ Eccentricity), and therefore exclude crowding effects (Levi, 2008). 


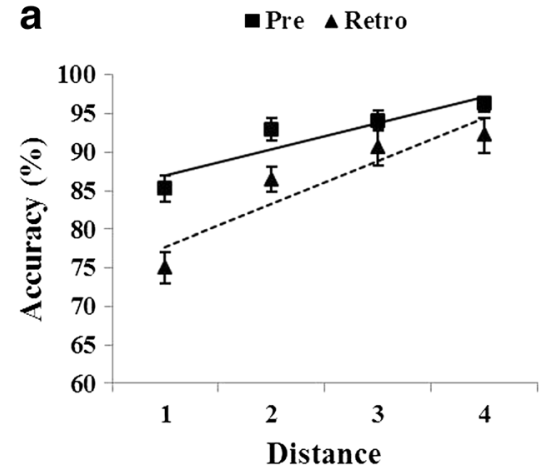

Fig. 2 Accuracy (a) and reaction times (b) by Distance and Cue Type from Experiment 1. Note that distance relates only to nonmatch trials. The regression lines for the pre-cue and retro-cue conditions are, respectively,

\section{Results}

Accuracy The analyses revealed a main effect of Cue Type $\left[F(1,18)=15.570, p=.001, \eta_{\mathrm{p}}{ }^{2}=.464\right]$, with higher accuracies in pre-cue than in retro-cue trials. Furthermore, a main of effect of Distance $\left[F(3,16)=14.917, p<.001, \eta_{\mathrm{p}}{ }^{2}=.737\right]$ was obtained. Finally, the analysis also revealed a significant Distance $\times$ Cue Type interaction $\left[F(3,16)=4.821, p=.014, \eta_{\mathrm{p}}{ }^{2}=\right.$ $.475]$. As in Experiment 1, our regression analyses revealed an accuracy increase with increasing distance for the retro-cue condition (slope $=4.98, S E=1.06), t(18)=4.68, p<.001$, $95 \% \mathrm{CI}=[2.38,7.58]$, and for the pre-cue condition (slope $=$ 2.67, $S E=0.56), t(18)=4.77, p<.001,95 \% \mathrm{CI}=[1.30$, 4.04]. This increase was larger in the retro-cue than in the precue condition (slope $=2.67, S E=0.56), t(18)=-2.3, p=.034$, $95 \% \mathrm{CI}=[-4.42,0.20]$. See Fig. 3 .

Reaction times The analyses revealed a significant effect of Distance $\left[F(3,16)=8.293, p=.001, \eta_{\mathrm{p}}{ }^{2}=.609\right]$. Although the Distance $\times$ Cue Type interaction did not reach significance at the $5 \%$ alpha level, it was marginally significant $[F(3,16)=$ $\left.2.981, p=.063, \eta_{\mathrm{p}}{ }^{2}=.359\right]$. Regression analyses showed that whereas reaction times remained stable in the retro-cue trials $($ slope $=-1.987, S E=5.07), t(18)=-0.391, p=.70,95 \% \mathrm{CI}=$

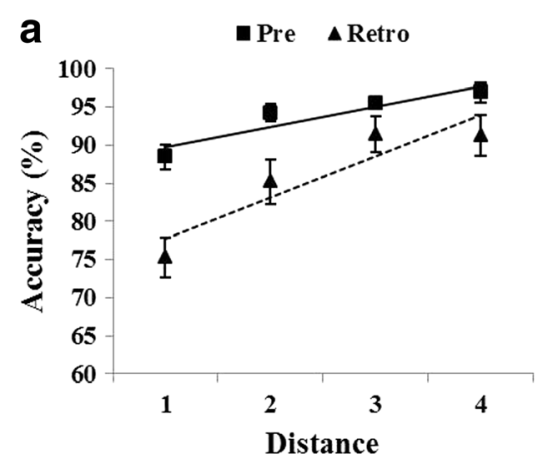

Fig. 3 Accuracy (a) and reaction times (b) by Distance and Cue Type from Experiment 2. Note that distance relates only to nonmatch trials. The regression lines for the pre-cue and retro-cue conditions are, respectively,

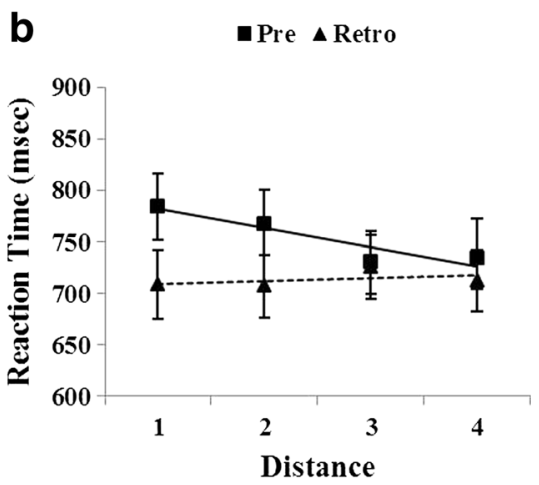

plotted in solid and dashed lines. In all cases, the error bars show the standard errors of the means

$[-14.41,10.43]$, they decreased with increasing distance in the pre-cue trials (slope $=-19.185, S E=3.86), t(18)=-4.97, p<$ $.001,95 \% \mathrm{CI}=[-28.63,-9.74]$.

When directly comparing the two experiments against one another, none of the within-subjects factors interacted with Experiment, nor did we observe a main effect of experiment (all $F \mathrm{~s}<1$ ) for either reaction times or accuracies.

\section{Discussion}

The purpose of Experiment 2 was, as in Experiment 1, to investigate how attention is distributed in mental space and whether this spatial attention gradient follows a pattern similar to that found in physical space. Crucially, the main objective was to test whether crowding effects were underlying our findings, rather than spatial attentional mechanisms per se. The key findings of Experiment 1 were replicated: Namely, performance gradually improved with increasing distractor distance in both mental and physical spaces. Moreover, no experiment effects were found, indicating that attentional selection mechanisms brought about the results in both experiments, rather than the effects being driven by crowding in Experiment 1.

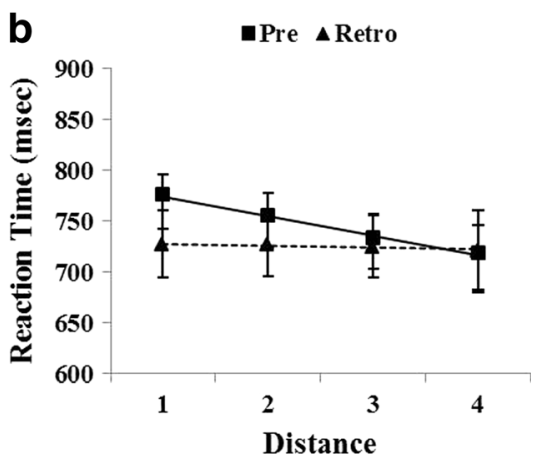

plotted in solid and dashed lines. In all cases, the error bars show the standard errors of the means 


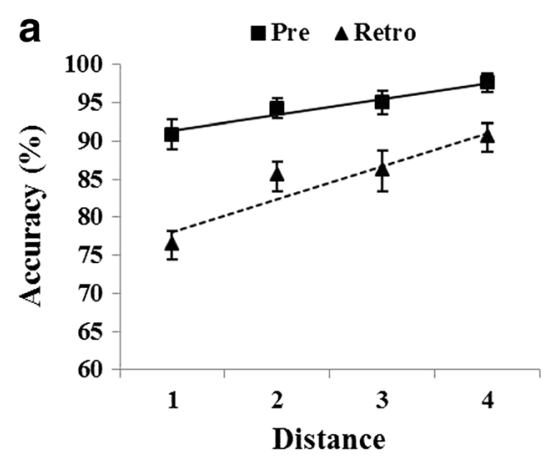

Fig. 4 Accuracy (a) and reaction times (b) by Distance and Cue Type from Experiment 3. Note that distance relates only to nonmatch trials. The regression lines for the pre-cue and retro-cue conditions are, respectively,

\section{Experiment 3}

Although the findings from Experiments 1 and 2 converge and generally confirm that spatial attention is distributed similarly in mental and physical spaces, as reflected by our accuracy results, it is not clear why reaction time measures would reveal a different pattern. Reaction times did not gradually decrease when retro-cues were administered, but remained stable and were overall faster than in the pre-cue condition. A possible explanation could be the difference in the durations of the interval between the moment of attentional selection and the response execution. Experiments 1 and 2 were designed such that the array and probe display were temporally aligned in the pre- and retro-cue conditions. Combined with the fact that the pre-cue was delivered before the array, and the retro-cue after the array, this implies that the time between attentional selection and the presentation of the probe lasted longer for the precue than for the retro-cue condition. In Experiment 3, the trial events were timed such that the intervals between attentional selection and the delivery of the probe were the same in these two conditions.

\section{Method}

Participants Twenty other Ghent University students (14 females, six males; $M=18.3$ years old, $S D=0.57$ ) participated for course credits.

Stimuli, design, and procedure All parameters were as in Experiment 2, except for the stimulus onsets in the trial sequence (see Fig. 1). The 1,500-2,500 ms intervals before and after the stimulus array, respectively, in the retrocue and pre-cue conditions of this experiment were removed. These manipulations enabled the pre-cue trials to closely match the retro-cue trials with respect to the timing of the critical attentional selection and probe events during the trial sequence.

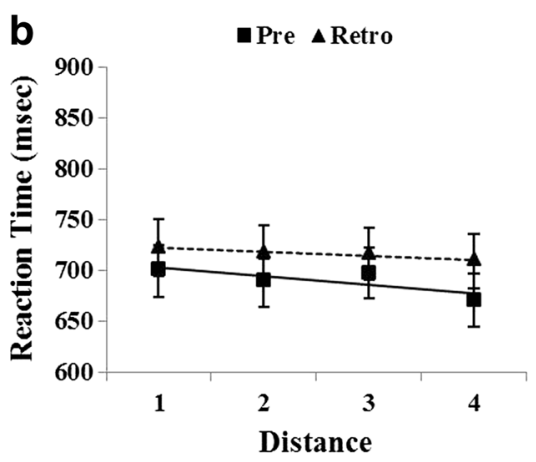

plotted in solid and dashed lines. In all cases, the error bars show the standard errors of the means

\section{Results}

Accuracy The analyses revealed a main effect of Cue Type $\left[F(1,19)=33.066, p<.001, \eta_{\mathrm{p}}{ }^{2}=.635\right]$, with higher accuracies in pre-cue than in retro-cue trials. Furthermore, a main of effect of Distance $\left[F(3,17)=25.749, p<.001, \eta_{\mathrm{p}}{ }^{2}=.820\right]$ was evidenced. To further examine the main effect of distance, a regression analysis was performed to test for linear effects of distance. The mean accuracies linearly increased with distance $($ slope $=3.24, S E=0.40), t(19)=8.102, p<.001,95 \% \mathrm{CI}=$ $[-2.41,4.08]$. The Distance $\times$ Cue Type interaction was marginally significant $\left[F(3,17)=2.834, p=.069, \eta_{\mathrm{p}}{ }^{2}=.333\right]$. Regression analyses revealed an accuracy increase with increasing distance for the retro-cue condition (slope $=4.32$, $S E=0.69), t(19)=6.20, p<.001,95 \% \mathrm{CI}=[2.62,6.02]$, and for the pre-cue condition ( slope $=2.09, S E=0.39), t(19)=$ $5.35, p<.001,95 \% \mathrm{CI}=[1.14,3.03]$, with the increase being larger in the retro-cue than in the pre-cue condition, $t(19)=-$ $2.87, p=.010,95 \% \mathrm{CI}=[-3.87,0.60]$. See Fig. 4 .

Reaction times The analyses revealed a marginally significant effect of Distance $\left[F(3,17)=2.923, p=.064, \eta_{\mathrm{p}}{ }^{2}=.340\right]$. However, neither the main effect of Cue Type $[F(1,19)=$ $\left.1.524, p=.23, \eta_{\mathrm{p}}{ }^{2}=.074\right]$ nor the Cue Type $\times$ Distance interaction $\left[F(3,17)=1.163, p=.35, \eta_{\mathrm{p}}{ }^{2}=.170\right]$ reached significance. Regression analyses showed that the slopes in the pre-cue and retro-cue conditions did not differ from each other, $t(19)=-0.861, p=.40,95 \% \mathrm{CI}=[-15.5,6.46]$.

\section{Discussion}

The present data resolve the remaining ambiguity from Experiments 1 and 2, in which the attentional distribution reflected the same distance-related pattern for the pre-cue and retro-cue conditions as measured by accuracy, but not by reaction times. In Experiment 3, with the same duration of the interval between attentional selection and probe, the difference in the reaction time patterns between the two cueing conditions disappeared. 


\section{General discussion}

Selective attention reduces the load on limited-capacity cognitive systems by not only filtering irrelevant distractors in the external visual space, but also by filtering irrelevant distractors within the internal mental space (Rowe \& Passingham, 2001). What has not been shown yet is whether the attentional selection of internal and external information relies on similar spatial mechanisms. It has been shown that orienting the focus of attention in mental space is similar to orienting attention in physical space (Griffin \& Nobre, 2003). However, these findings do not clarify how the unattended information at different locations outside the focus of attention is dealt with. Are unattended locations in mental space merely discarded from memory, regardless of their location? Or are they instead subjected to a spatial gradient based on their relative distance from the focus of attention, very much like what is usually observed for spatial attention to physical space?

Across three experiments, we consistently found that selective attention modulates the strength of the internal representations in mental space in the same way that it operates in physical space. Crucially, our findings demonstrate that attention is distributed across unattended locations in mental space with distributional characteristics similar to those found for attention in physical space. More precisely, performance is marked by a gradual improvement when the focus of attention is probed with a distractor item that originated from more remote locations in either a mentally represented or a physically present stimulus array.

Without additional assumptions, the observation that performance gradually improved with increasing distractor distance is not compatible with spotlight (Posner, 1980), zoom lens (Eriksen \& Yeh, 1985), or center-surround (Tsotsos et al., 1995) models. In contrast, it is completely in line with predictions from the gradient model (Downing \& Pinker, 1985).

Our results can be naturally accommodated within the resource allocation theory of working memory (Bays \& Husain, 2008), since this theory assumes flexible assignment of resources to the items to be remembered. Thus far, research within this framework has primarily focused on the allocation of resources as a function of the number of items to be remembered; however, it has recently also been established that an attended item receives more resources than an unattended item (Pertzov, Bays, Joseph, \& Husain, 2013). Our findings add to this literature by providing strong indications that the resources that are allocated to the unattended items are distributed as a function of the distance from the attended item. This view has also been implemented in other computational models (e.g., Kahana \& Sekuler, 2002; Sederberg, Miller, Howard, \& Kahana, 2010).

It is noteworthy that the gradual performance increase was more pronounced in the retro-cue than in the pre-cue condition. One could argue that this is indicative of different selection mechanisms. However, this is unlikely, given that the distribution patterns of attention in mental and physical space match in terms of their shapes. Instead, the slope differences may emerge due to differences in visual resolution between mental representations and perceptual stimuli. As visual resolution decays in memory, the confusability for distances near the focus of attention may be higher in mental than in physical space (Ma, Husain, \& Bays, 2014).

In summary, the present data suggest that the distribution of attention follows similar patterns in physical and mental space, with information close to the focus attention inducing more confusion than more distant information. Whether this means that attentional selection involves the same system in physical and mental space (e.g., Awh \& Jonides, 2001) or, alternatively, engages independent systems (e.g., Hedge, Oberauer, \& Leonards, 2015) with similar properties is matter for further research.

\section{References}

Awh, E., \& Jonides, J. (2001). Overlapping mechanisms of attention and spatial working memory. Trends in Cognitive Sciences, 5, 119-126. doi:10.1016/S1364-6613(00)01593-X

Bays, P. M., \& Husain, M. (2008). Dynamic shifts of limited working memory resources in human vision. Science, 321, 851-854. doi:10. 1126/science. 1158023

Downing, C. J., \& Pinker, S. (1985). The spatial structure of visual attention. In Attention and Performance XI. Hillsdale, NJ: Erlbaum.

Eriksen, C. W., \& Yeh, Y. (1985). Allocation of attention in the visual field. Journal of Experimental Psychology: Human Perception and Performance, 11, 583-597. doi:10.1037/0096-1523.11.5.583

Gazzaley, A., \& Nobre, A. C. (2012). Top-down modulation: Bridging selective attention and working memory. Trends in Cognitive Sciences, 16, 129-135. doi:10.1016/j.tics.2011.11.014

Griffin, I. C., \& Nobre, A. C. (2003). Orienting attention to locations in internal representations. Journal of Cognitive Neuroscience, 15, 1176-1194. doi:10.1162/089892903322598139

Hedge, C., Oberauer, K., \& Leonards, U. (2015). Selection in spatial working memory is independent of perceptual selective attention, but they interact in a shared spatial priority map. Attention, Perception, \& Psychophysics, 77, 2653-2668. doi:10.3758/ s13414-015-0976-4

Hopf, J. M., Boehler, C. N., Luck, S. J., Tsotsos, J. K., Heinze, H. J., \& Schoenfeld, M. A. (2006). Direct neurophysiological evidence for spatial suppression surrounding the focus of attention in vision. Proceedings of the National Academy of Sciences, 103, 10531058. doi:10.1073/pnas.0507746103

Kahana, M. J., \& Sekuler, R. (2002). Recognizing spatial patterns: A noisy exemplar approach. Vision Research, 42, 2177-2192. doi:10. 1016/S0042-6989(02)00118-9

Kiyonaga, A., \& Egner, T. (2013). Working memory as internal attention: Toward an integrative account of internal and external selection processes. Psychonomic Bulletin \& Review, 20, 228-242. doi:10. 3758/s13423-012-0359-y

Levi, D. M. (2008). Crowding-An essential bottleneck for object recognition: A mini-review. Vision Research, 48, 635-654. doi:10. 1016/j.visres.2007.12.009 
Lorch, R. F., \& Myers, J. L. (1990). Regression analyses of repeated measures data in cognitive research. Journal of Experimental Psychology: Learning, Memory, and Cognition, 16, 149-157. doi: 10.1037/0278-7393.16.1.149

Ma, W. J., Husain, M., \& Bays, P. M. (2014). Changing concepts of working memory. Nature Neuroscience, 17, 347-356. doi:10.1038/nn.3655

Oberauer, K. (2009). Design for a working memory. In B. H. Ross (Ed.), The psychology of learning and motivation: Advances in research and theory (Vol. 51, pp. 45-100). San Diego, CA: Elsevier Academic Press. doi:10.1016/S0079-7421(09)51002-X

Pertzov, Y., Bays, P. M., Joseph, S., \& Husain, M. (2013). Rapid forgetting prevented by retrospective attention cues. Journal of Experimental Psychology: Human Perception and Performance, 39, 1224-1231. doi:10.1037/a0030947

Posner, M. I. (1980). Orienting of attention. Quarterly Journal of Experimental Psychology, 32, 3-25. doi:10.1080/00335558008248231
Postle, B. R. (2006). Working memory as an emergent property of the mind and brain. Neuroscience, 139, 23-38. doi:10.1016/j. neuroscience.2005.06.005

Rowe, J. B., \& Passingham, R. E. (2001). Working memory for location and time: Activity in prefrontal area 46 relates to selection rather than maintenance in memory. NeuroImage, 14, 77-86. doi:10.1006/ nimg.2001.0784

Sederberg, P. B., Miller, J. F., Howard, M. W., \& Kahana, M. J. (2010). The temporal contiguity effect predicts episodic memory performance. Memory \& Cognition, 38, 689-699. doi:10.3758/MC.38.6.689

Störmer, V. S., \& Alvarez, G. A. (2014). Feature-based attention elicits surround suppression in feature space. Current Biology, 24, 19851988. doi:10.1016/j.cub.2014.07.030

Tsotsos, J. K., Culhane, S. M., Winky, Y. W. K., Yuzhong, L., Davis, N., \& Fernando, N. (1995). Artificial intelligence modeling visual attention. Artificial Intelligence, 3702, 507-545. 